\title{
Nuclear Star Clusters (Nuclei) in Spirals and Connection to Supermassive Black Holes
}

\author{
Roeland P. van der Marel ${ }^{1}$, Joern Rossa ${ }^{2}$, Carl Jakob Walcher ${ }^{3}$, \\ Torsten Boeker ${ }^{4}$, Luis C. $\mathrm{Ho}^{5}$, \\ Hans-Walter Rix ${ }^{6}$ and Joseph C. Shields ${ }^{7}$ \\ ${ }^{1}$ STScI, 3700 San Martin Drive, Baltimore, MD 21218, USA \\ ${ }^{2}$ Dept. of Astronomy, University of Florida, Gainesville, FL 32611, USA \\ ${ }^{3}$ Laboratoire d'Astrophysique de Marseille, F-13376 Marseille Cedex 12, France \\ ${ }^{4}$ European Space Agency, Department RSSD, 2200 AG Noordwijk, The Netherlands \\ ${ }^{5}$ Carnegie Institution of Washington, 813 Santa Barbara Street, Pasadena, CA 91101, USA \\ ${ }^{6}$ Max-Planck-Institut für Astronomie, Königstuhl 17, D-69117 Heidelberg, Germany \\ ${ }^{7}$ Dept. of Physics and Astronomy, Ohio University, Athens, OH 45701, USA
}

\begin{abstract}
HST observations have revealed that compact sources exist at the centers of many, maybe even most, galaxies across the Hubble sequence. These sources are called "nuclei" or also "nuclear star clusters" (NCs), given that their structural properties and position in the fundamental plane are similar to those of globular clusters. Interest in NCs increased recently due to the independent and contemporaneous finding of three groups (Rossa et al. for spiral galaxies; Wehner \& Harris for dE galaxies; and Côté et al. for elliptical galaxies) that NC masses obey similar scaling relationships with host galaxy properties as do supermassive black holes. Here we summarize the results of our group on NCs in spiral galaxies. We discuss the implications for our understanding of the formation and evolution of NCs and their possible connection to supermassive black holes.
\end{abstract}

\section{Introduction}

High spatial resolution observations with the Hubble Space Telescope (HST) have revealed that many galaxies have a compact source in their very center. These "nuclei" have structural properties that are similar to those of globular clusters (see below), and they are therefore also called "nuclear star clusters" (NCs). Figure 1a shows the nearby Sd galaxy NGC 300 as an example. This galaxy has a NC, which is easily identified as a separate component because of the marked upturn in the surface brightness profile at small radii (Figure 1b). Boeker et al. (2002, 2004) observed a sample of 77 late-type spiral galaxies with HST/WFPC2 and found NCs in $77 \%$ of the sample. Fits to the two-dimensional images yield the effective radii and luminosities of the NCs, which for late-type spirals have median values $r_{\text {eff }}=3.5 \mathrm{pc}$ and $L_{I}=10^{6.2} L_{\odot}$.

The study of NCs in late-type spirals is facilitated by the faintness of an underlying bulge/spheroid component. The same is true for the study of nuclei in $\mathrm{dE}$ galaxies. However, NCs are also found in other galaxy types. Carollo et al. $(1997,1998)$ report a detection frequency of 50-60\% in early-type spirals using HST/WFPC2 imaging. Côté et al. (2006) report the detection of nuclei in $66-82 \%$ of intermediate-luminosity Virgo cluster elliptical galaxies (but no detections in the most luminous giant ellipticals) using HST/ACS imaging. The detection of NCs does become progressively more difficult as the density of the underlying spheroid increases, which is a problem especially for elliptical 

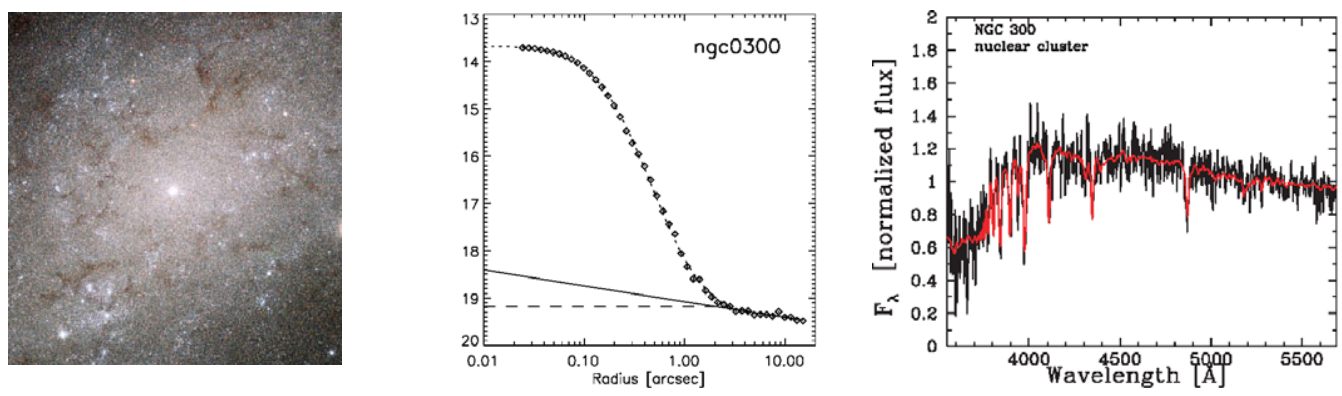

Figure 1. (a) HST/ACS color composite image of the central $1.2 \times 1.2 \mathrm{kpc}$ of the Sd galaxy NGC 300 at $D \approx 1.8 \mathrm{Mpc}$ (from the observations of Bresolin et al. 2005). A prominent nuclear star cluster $(\mathrm{NC})$ resides at the center of the galaxy. (b) $I$-band surface brightness profile in $\mathrm{mag} / \operatorname{arcsec}^{2}$ for the central $\sim 10^{\prime \prime}=87 \mathrm{pc}$, as determined from HST/WFPC2 data of the same galaxy (Boeker et al. 2002). The NC is evident as a marked upturn in the brightness profile, as compared to inward extrapolations (solid, dashed lines) of the the brightness profile at large radii. King-model fits to the two-dimensional image imply a NC luminosity $L_{I}=10^{6.2} L_{\odot}$ and effective radius $r_{\text {eff }}=2.9 \mathrm{pc}$ (Boeker et al. 2004). (c) HST/STIS spectrum of the NC in NGC 300 (from Rossa et al. 2006). The red curve shows the best spectral population fit, which has $Z=0.004$, $A_{V}=0.4$, a luminosity-weighted mean $\langle\log ($ age $/ \mathrm{Gyr})\rangle=8.63$ and $M / L_{B}=0.51 M_{\odot} / L_{B, \odot}$. The implied NC mass is $M=10^{5.7} M_{\odot}$.

galaxies with steep cusps. The inferred NC characteristics then depend sensitively on the assumed spheroid brightness profile (Lauer et al. 2006; Ferrarese et al. 2006b).

\section{Spectroscopy of Nuclear Star Clusters in Spiral Galaxies}

$\mathrm{NCs} /$ nuclei are a common component of galaxies across the Hubble sequence. It is therefore important to have observational knowledge of their stellar populations, ages, and masses. We pursued a spectroscopic program to measure these quantities for spiral galaxies. We used the UVES echelle spectrograph on the VLT to obtain spectra of the NCs in nine late-type spirals (Walcher et al. 2005, 2006). The high spectral resolution of these data allows the measurement of the $\mathrm{NC}$ velocity dispersions from the near-IR Ca II triplet line broadening. The results fall in the range $\sigma=13-34 \mathrm{~km} / \mathrm{s}$. When interpreted with spherically symmetric dynamical models tailored to fit the HST surface brightness profiles, these measurements yield the NC masses. Figure 2 a shows the NC properties in a face-on fundamental plane projection. The NCs fall on a high-mass extension of the globular cluster sequence.

The VLT ground-based spatial resolution contaminates the NC light with that of the underlying galaxy. This is manageable in the latest-type spirals, but makes it difficult to study NCs in earlier-type spirals. We therefore also pursued a program with HST/STIS of 40 spiral galaxies of various Hubble types (Rossa et al. 2006). These spectra have the advantage of higher spatial resolution that can cleanly separate the NC light from the underlying galaxy light. However, the $S / N$ and spectral resolution are much lower than for the VLT spectra, which makes it impossible to infer the NC kinematics.

We fitted the VLT and the STIS spectra using a non-negative weighted linear sum of Bruzual \& Charlot (2003) single-age templates with different ages. This yields for each NC the extinction, metallicity, star formation history, luminosity-weighted mean $\log ($ age), and $M / L$. Figure 1c shows the STIS spectrum of NGC 300 to illustrate this approach. The $M / L$ inferred from the spectral population fitting can be combined with the luminosity inferred from HST imaging to yield the NC mass. For the nine NCs with VLT spectra, we find that the $M / L$ and $M$ values inferred from dynamical modeling and spectral population fitting are in good agreement. 

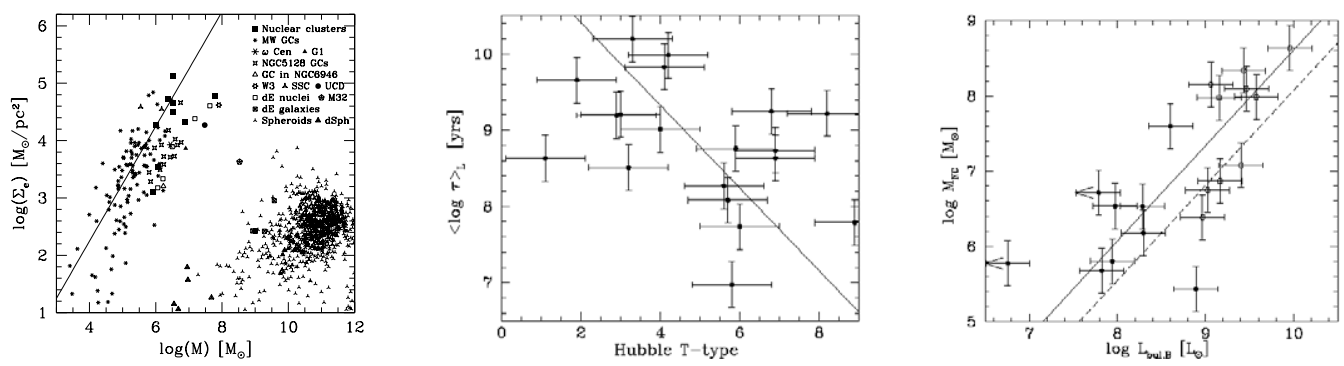

Figure 2. (a) Mean projected mass density inside $r_{\text {eff }}$ versus total mass, for different types of stellar systems as labeled in the plot (from Walcher et al. 2005). Elliptical galaxies and bulges are found on the right side of the plot, which presents an approximately face-on view of their fundamental plane. Globular clusters are found on the left, roughly along a locus of constant $r_{\text {eff }} \approx 3 \mathrm{pc}$ (solid line). The nuclear star clusters (NCs) in spiral galaxies (solid squares) lie at the high-mass end of the globular cluster sequence, as do the nuclei of dE galaxies. $(b)$ Luminosity-weighted mean $\log ($ age/Gyr) versus Hubble type. NCs in late-type spiral galaxies tend to have younger populations than those in early-type spiral galaxies. (c) NC mass versus $B$-band luminosity of the host galaxy bulge. Open symbols denote early-type spirals and solid symbols denote late-type spirals. Galaxies with more luminous bulges tend to have more massive NCs. Solid lines are least squares fits to the data points. Panels (b,c) are from Rossa et al. (2006), and are based on stellar population fits to HST/STIS spectra such as those shown in Figure 1c. The dashed line in the right panel indicates the relation between the supermassive black hole mass and bulge luminosity for galaxies studied by Marconi \& Hunt (2003).

The NCs have mixed-age populations, with single-age fits generally ruled out by $\chi^{2}$ statistics. This is supported by an analysis of line-strength indices measured from the VLT spectra. Approximately half of the NCs in the STIS sample contain a population younger than 1 Gyr. The luminosity-weighted ages range from 10 Myrs to 10 Gyrs. The NCs in late-type spirals tend to have younger luminosity-weighted mean ages than those in earlytype spirals (Figure $2 \mathrm{~b}$ ). But even when a young population dominates the light, we find that there is generally an underlying older population that contains most of the mass.

Carollo et al. (2002) found that NC luminosities in early-type spirals are on average brighter than those in late-type spirals. Our results show that this is true for NC masses as well: they correlate loosely with total galaxy luminosity. Figure $2 \mathrm{c}$ shows that there is an even stronger correlation with the luminosity of the host galaxy bulge. This correlation has the same slope as the well-known correlation between supermassive black hole (BH) mass and bulge luminosity (dashed line in Figure 2c).

\section{Discussion}

The results of the spectroscopic analysis provide insight into several important questions.

What do we know about NC formation? NCs have similar structural properties as globular clusters. This suggests a commonality in the physical processes that shaped them, despite their different environments. By contrast, bulges follow very different scaling relationships, indicating that their formation was probably governed by different physical processes. NCs generally have underlying old stellar populations, suggesting that they may have formed early in the life of the galaxy. Moreover, NC formation must be efficient, because NCs/nuclei are a common feature of galaxies. However, there must be circumstances that prevent $\mathrm{NC}$ formation in some fraction of galaxies.

How do NCs and the galaxies they reside in evolve? NCs have had repeated star formation episodes. So they grow in mass as time passes, due to supply of new gas to the galaxy center through secular evolution processes (e.g., bar torques). NCs in later-type galaxies on average have younger populations, probably due to their larger gas supply. 
By contrast, nuclei in dEs have predominantly old populations (Geha, Guhathakurta \& van der Marel 2003), probably reflecting their general lack of gas. In fact, dEs may have formed through transformation of late-type spirals (Moore, Lake \& Katz 1998), so dE nuclei may be the more evolved counterparts of the NCs in spirals. NCs can also cause secular evolution, given that their masses can be sufficient to drive the destruction of bars and the formation of pseudo-bulges (Carollo 1999; Kormendy \& Kennicut 2004).

What is the relation between NCs and supermassive BHs? NC masses scale with host galaxy properties similarly as BH masses. Contemporaneously with our work on spirals (Rossa et al. 2006), this was independently shown also for the nuclei in dE galaxies (Wehner \& Harris 2006) and the nuclei in intermediate-luminosity elliptical galaxies (Côté et al. 2006). In all cases the NC masses are a few times larger than BH masses that one would predict based on established relations with $L_{\text {bulge }}$ (see, e.g., Figure 2c) or $\sigma$. Ferrarese et al. (2006a) showed that better agreement is obtained when host galaxy mass is used as the independent variable. Also, BHs are typically measured in galaxies more massive than a few times $10^{10} M_{\odot}$, whereas NCs are typically detected in less massive galaxies. This latter finding could indicate that they are different manifestations of a single "Compact Massive Object" class. But it could also be a selection effect; e.g., the dwarf Seyfert NGC 4395 has both a BH and a NC (Filippenko \& Ho 2003). The implications of all these findings are discussed in detail in each of the cited papers, including the interesting possibility that BHs and NCs are somehow causally or evolutionarily connected. However, this does not necessarily follow from the data. McLaughlin et al. (2006) discussed a feedback mechanism that can account for all observed relations without invoking any direct connection between BHs and NCs. As of yet, robust insights into the true BH-NC connection are still mostly missing.

\section{Acknowledgements}

Support for HST proposals \#9070 and \#9783 was provided by NASA through a grant from STScI, which is operated by AURA, Inc., under NASA contract NAS 5-26555.

\section{References}

Boeker, T., Laine, S., van der Marel, R. P., Sarzi, M., Rix, H.W., et al. 2002, AJ, 123, 1389

Boeker, T., Sarzi, M., McLaughlin, D. E., van der Marel, R. P., et al. 2004, AJ, 127, 105

Bresolin, F., Pietrzynski, G., Gieren, W., \& Kudritzki, R.-P. 2005, ApJ, 634, 1020

Bruzual, G., \& Charlot, S. 2003, MNRAS, 344, 1000

Carollo, C. M., Stiavelli, M., de Zeeuw, P. T., \& Mack, J. 1997, AJ, 114, 2366

Carollo, C. M., Stiavelli, M., \& Mack, J. 1998, AJ, 116, 68

Carollo, C. M. 1999, ApJ, 523, 566

Carollo, C. M., Stiavelli, M., Seigar, M., de Zeeuw, P. T., \& Dejonghe, H. 2002, AJ, 123, 159

Côté, P., et al. 2006, ApJS, 165, 57

Ferrarese, L., et al. 2006a, ApJ, 644, L21

Ferrarese, L., Côté, P., Blakeslee, J. P., Mei, S., Merritt, D., West, M. 2006b [astro-ph/0612139]

Filippenko, A. V., \& Ho, L. C. 2003, ApJ, 588, L13

Geha, M., Guhathakurta, P., \& van der Marel, R. P. 2003, AJ, 126, 1794

Kormendy, J., \& Kennicutt, R. C., Jr. 2004, ARA\&A, 42, 603

Lauer, T., et al. 2006, ApJ, submitted [astro-ph/0609762]

Marconi, A., \& Hunt, L. K. 2003, ApJ, 589, L21

McLaughlin, D. E., King, A. R., \& Nayakshin, S. 2006, ApJ, 650, L37

Moore, B., Lake, G., \& Katz, N. 1998, ApJ, 495, 139

Rossa, J., van der Marel, R. P., Boeker, T., Gerssen, J., Ho, L. C., et al. 2006, AJ, 132, 1074

Walcher, C. J., van der Marel, R. P., McLaughlin, D. E., Rix, H.-W., et al. 2005, ApJ, 618, 237

Walcher, C. J., Boeker, T., Charlot, S., Ho, L. C., Rix, H, Rossa, J., et al. 2006, ApJ, 649, 692

Wehner, E.H., \& Harris, W. 2006, ApJ, 644, L17 


\section{Discussion}

DE JONG: What IMF did you assume in the comparison of the stellar population and dynamical masses of the NCs?

VAN DER MAREL: We used the Bruzual-Charlot (2003) stellar population models with the Chabrier (2003) IMF and a cut-off at $0.1 M_{\odot}$. The fact that our stellar population and dynamical masses are in good agreement provides support to the hypothesis that this is a reasonable assumption.

PeLetier: Are the NCs in your early-type spirals resolved? In our NICMOS study (Balcells et al. 2003, 2006) we find that they are generally unresolved. Also, we have done simulations showing that in early-type spirals their detection threshold is a strong function of bulge luminosity, implying that it might be hard to establish whether there is a correlation between NC mass and galaxy type.

VAN DER MAREL: Our observations show that NCs in late-type spirals are generally resolved (Boeker et al. 2004). We have not done imaging of early-type spirals ourselves. However, Carollo and coworkers have observed many early-type spirals and do often report the NCs to be resolved. Since the sizes of the NCs are often close to the resolution limit, it does help to observe in the optical instead of the near-IR. This may be a partial explanation for the apparent difference with your result. Characterization of NCs is indeed complicated in the presence of a steep underlying bulge, so I agree that apparent correlations should be interpreted with care. However, it is remarkable that a similar correlation between NC mass and host galaxy properties has been found independently for spirals, dE galaxies, and intermediate-luminosity elliptical galaxies. This strongly suggests that the inferred correlations are not merely driven by systematic uncertainties in image decompositions, but have a physical basis.

BALCELLS: We found NCs in $\sim 60 \%$ of S0-Sb galaxy bulges. However, we find that their masses do not fall on the extrapolation of the $M_{\mathrm{BH}^{-}} \sigma$ correlation. As eluded to by Reynier Peletier, this cast doubts on the possible connection between BHs and NCs. My question is, how much underlying disk contamination do you have in the VLT/UVES spectra, and how did you deal with it?

VAN DER MAREL: We applied a background subtraction to both the VLT/UVES spectra and the HST/STIS spectra, using data along the slit on either side of the NC. The residual contamination is managable, for VLT/UVES because we only observed late-type spirals and for the HST/STIS spectra because of the high spatial resolution. But to transform the inferred $\mathrm{M} / \mathrm{L}$ into a NC mass one needs to know the NC luminosity, which requires a decomposition of two-dimensional HST images. For spirals we have used the results of Carollo et al. (1998). This is indeed an important source of uncertainty, especially for bulges and ellipticals with steep cusps.

REnZINI: Ferrarese et al. (2006a) suggest that NCs may be failed BHs. Yet I believe we know of one case in which there is both a $\mathrm{NC}$ and a $\mathrm{BH}$, namely the Milky Way.

VAN DER MAREL: Indeed, and the dwarf Seyfert galaxy NGC 4395 is another example of this. These examples and other independent arguments suggest that it is too early to conclude that BHs and NCs are somehow causally or evolutionarily connected, although that certainly is an interesting possibility. 